Ardonne, Eddy; Bouwknegt, Peter G.; Guruswamy, Sathya; Schoutens, Kareljan K-matrices for non-Abelian quantum Hall states Physical Review B, 2000; 61(15):1029810302

(C) 2000 American Physical Society

http://link.aps.org.proxy.library.adelaide.edu.au/doi/10.1103/PhysRevB.61.10298

PERMISSIONS

http://publish.aps.org/authors/transfer-of-copyright-agreement

"The author(s), and in the case of a Work Made For Hire, as defined in the U.S.

Copyright Act, 17 U.S.C.

$\S 101$, the employer named [below], shall have the following rights (the "Author Rights"):

$[\ldots]$

3. The right to use all or part of the Article, including the APS-prepared version without revision or modification, on the author(s)' web home page or employer's website and to make copies of all or part of the Article, including the APS-prepared version without revision or modification, for the author(s)' and/or the employer's use for educational or research purposes."

$21^{\text {th }}$ March 2013 


\title{
$K$-matrices for non-Abelian quantum Hall states
}

\author{
Eddy Ardonne \\ Institute for Theoretical Physics, University of Amsterdam, Valckenierstraat 65, 1018 XE Amsterdam, The Netherlands \\ Peter Bouwknegt \\ Department of Physics and Mathematical Physics, University of Adelaide, Adelaide, SA 5005, Australia \\ Sathya Guruswamy and Kareljan Schoutens \\ Institute for Theoretical Physics, University of Amsterdam, Valckenierstraat 65, 1018 XE Amsterdam, The Netherlands
}

(Received 27 August 1999)

\begin{abstract}
Two fundamental aspects of so-called non-Abelian quantum Hall states (the $q$-Pfaffian states and more general) are a (generalized) pairing of the participating electrons and the non-Abelian statistics of the quasihole excitations. In this paper, we show that these two aspects are linked by a duality relation, which can be made manifest by considering the $K$-matrices that describe the exclusion statistics of the fundamental excitations in these systems.
\end{abstract}

\section{INTRODUCTION}

In the description of low-energy excitations over Abelian fractional quantum Hall $(\mathrm{FQH})$ states, an important role is played by the so-called $K$-matrices, which characterize the topological order of the FQH state (see Ref. 1 for a review). These $K$-matrices act as parameters in Landau-GinzburgChern-Simons (LGCS) field theories for bulk excitations and in the chiral conformal field theories (CFT's) that describe excitations at the edge. At the same time, the $K$-matrices provide the parameters for the fractional exclusion statistics (in the sense of Haldane ${ }^{2}$ ) of the edge excitations over the FQH states. For a simple example of this, consider the Laughlin FQH state at filling fraction $\nu=1 / m$, with $K$-matrix equal to the number $m$. Following the analysis in Ref. 3, one finds that the parameters $g_{e}$ and $g_{\phi}$ that characterize the exclusion statistics of the edge electrons (of charge $Q=-e$ ) and edge quasiholes $(Q=+e / m)$, respectively, are given by

$$
g_{e}=\mathbf{K}=m, \quad g_{\phi}=\mathbf{K}^{-1}=\frac{1}{m} .
$$

In this paper, we shall denote these and similar parameters by $\mathbf{K}_{e}$ and $\mathbf{K}_{\phi}$, respectively.

The $(e, \phi)$ basis for edge excitations is natural in view of the following statements about duality and completeness. The particle-hole duality between the edge electron and quasihole excitations is expressed through the relation $\mathbf{K}_{\phi}$ $=\mathbf{K}_{e}^{-1}$ and through the absence of mutual exclusion statistics between the two. It leads to the following relation between the one-particle distribution functions $n_{e}(\epsilon)$ and $n_{\phi}(\epsilon)$ :

$$
m n_{e}(\boldsymbol{\epsilon})=1-\frac{1}{m} n_{\phi}\left(-\frac{\boldsymbol{\epsilon}}{m}\right) .
$$

In physical terms, the duality implies that the absence of edge electrons with energies $\epsilon<0$ is equivalent to the presence of edge quasiholes with positive energies, and vice versa. By the completeness of the $(e, \phi)$ basis we mean that the collection of all multi-e, multi- $\phi$ states spans a basis of the chiral Hilbert space of all edge excitations. In mathematical terms, this is expressed by a formula that expresses the partition sum for the edge excitations as a so-called universal chiral partition function (UCPF) (see, e.g., Ref. 4 and references therein) based on the matrix

$$
\mathbf{K}_{e} \oplus \mathbf{K}_{\phi}=\left(\begin{array}{cc}
m & 0 \\
0 & 1 / m
\end{array}\right) .
$$

We refer to Ref. 3 for an extensive discussion of these results, and to Ref. 5 for an extension to (Abelian) FQH states with more general $n \times n K$-matrices.

Turning our attention to non-Abelian quantum Hall states, we observe that the chiral CFT's for the edge excitations are not free-boson theories. This implies that for these states the notion of a $K$-matrix needs to be generalized. In this paper, we shall show that the exclusion statistics of the fundamental edge "quasihole", and "electron" excitations over nonAbelian quantum Hall states give rise to matrices that are closely analogous to the $K$-matrices in the Abelian case, and we therefore refer to these matrices as the $K$-matrices for non-Abelian quantum Hall states. We present results for the $q$-Pfaffian state, ${ }^{6}$ for the so-called parafermionic quantum Hall states, ${ }^{7}$ and for the non-Abelian spin-singlet states recently proposed in Ref. 8. A more detailed account, including explicit derivations of the results presented here, will be given elsewhere.

\section{PFAFFIAN QUANTUM HALL STATES FOR SPINLESS ELECTRONS}

The so-called $q$-Pfaffian quantum Hall states at filling fraction $\nu=1 / q$ were proposed in 1991 by Moore and $\operatorname{Read}^{6}$ and have been studied in considerable detail. ${ }^{10-12}$ The charged spectrum contains fundamental quasiholes of charge $e / 2 q$ and electron-type excitations with charge $-e$ and fermionic braid statistics. The edge CFT can be written in terms of a single chiral boson and real (Majorana) fermion, leading 
to a central charge $c=\frac{3}{2}$. The exclusion statistics of the edge quasiholes were studied in Refs. 13, 14. In Ref. 14, it was found that the thermodynamics of the edge quasihole can be described by the equations

$$
\frac{\lambda_{0}-1}{\lambda_{0}} \lambda_{0} \lambda^{-1 / 2}=1, \quad \frac{\lambda-1}{\lambda} \lambda_{0}^{-1 / 2} \lambda^{(q+1) / 4 q}=x,
$$

with $x=e^{\beta\left(\mu_{\phi}-\epsilon\right)}$. Comparing this with the general form of the Isakov-Ouvry-Wu (IOW) equations for particles with exclusion statistics matrix $\mathbf{K},{ }^{15}$

$$
\left(\frac{\lambda_{a}-1}{\lambda_{a}}\right) \prod_{b} \lambda_{b}^{K_{a b}}=x_{a},
$$

one identifies

$$
\mathbf{K}_{\phi}=\left(\begin{array}{cc}
1 & -\frac{1}{2} \\
-\frac{1}{2} & \frac{q+1}{4 q}
\end{array}\right)
$$

as the statistics matrix for particles $\left(\phi_{0}, \phi\right)$, where $\phi$ is the edge quasihole of charge $e / 2 q$. The other particle $\phi_{0}$ does not carry any charge or energy and is called a pseudoparticle. The presence of this particle accounts for the nonAbelian statistics of the physical particle $\phi .{ }^{14,16}$ Eliminating $\lambda_{0}$ from Eqs. (2.1) gives

$$
(\lambda-1)\left(\lambda^{1 / 2}-1\right)=x^{2} \lambda^{(3 q-1) / 2 q},
$$

in agreement with Ref. 13.

The duality between the $\phi$ and $e$ excitations over the $q$-Pfaffian state was first discussed in Ref. 13, where it was also shown how the correct spectrum of the edge CFT is reconstructed using the $e$ and $\phi$ quanta. Here we present a discussion at the level of $K$-matrices where, as in Eq. (1.1), the dual sector is reached by inverting the matrix $\mathbf{K}_{\phi}$ of Eq. (2.3).

Starting from the IOW equations (2.2) and denoting by $\lambda_{a}^{\prime}$ and $x_{a}^{\prime}$ the quantities corresponding to $\mathbf{K}^{\prime}=\mathbf{K}^{-1}$, we have the correspondence ${ }^{9}$

$$
\lambda_{a}^{\prime}=\frac{\lambda_{a}}{\lambda_{a}-1}, \quad x_{a}^{\prime}=\prod_{b} x_{b}^{-K_{a b}^{-1}},
$$

which leads, among other things, directly to the (appropriate generalization of) Eq. (1.2).

For the $q$-Pfaffian state, we define the $K$-matrix for the electron sector ( $e$ sector) to be the inverse of $\mathbf{K}_{\phi}$,

$$
\mathbf{K}_{e}=\mathbf{K}_{\phi}^{-1}=\left(\begin{array}{cc}
q+1 & 2 q \\
2 q & 4 q
\end{array}\right) .
$$

Inspecting the right-hand sides of the duality-transformed IOW equations, we find $x_{1}^{\prime}=y$ and $x_{2}^{\prime}=y^{2}$, with $y$ $=e^{\beta\left(\mu_{e}-\epsilon^{\prime}\right)}$, where $\epsilon^{\prime}=-2 q \epsilon$ and $\mu_{e}=-2 q \mu_{\phi}$ (i.e., $y$ $\left.=x^{-2 q}\right)$, indicating that the two particles in the $e$ sector carry charge $Q=-e$ and $Q=-2 e$, respectively. We shall denote these particles by $\Psi_{1}$ and $\Psi_{2}$. The first particle is quickly identified with the edge electron, with self-exclusion parameter equal to $q+1$. The presence of a "composite" particle
$\Psi_{2}$ of charge $-2 e$ has its origin in the fundamental electron pairing that is implied by the form of the Pfaffian wave function.

As before, there is no mutual exclusion statistics between the $e$ and $\phi$ sectors. In Ref. 9, we show that the conformal characters of the edge CFT can be cast in the UCPE form with matrix $\mathbf{K}_{e} \oplus \mathbf{K}_{\phi}$. The value $c=\frac{3}{2}$ of the central charge follows as a direct consequence. ${ }^{16}$

We remark that the usual relations between the charge vectors $\mathbf{Q}_{e}^{T}=(-e,-2 e), \mathbf{Q}_{\phi}^{T}=(0, e / 2 q)$, the matrices $\mathbf{K}_{e}$, $\mathbf{K}_{\phi}$, and the filling fraction $\nu$ are satisfied in this nonAbelian context:

$$
\begin{gathered}
\mathbf{K}_{e}=\mathbf{K}_{\phi}^{-1}, \quad \mathbf{Q}_{e}=-\mathbf{K}_{\phi}^{-1} \cdot \mathbf{Q}_{\phi}, \\
\nu e^{2}=\mathbf{Q}_{\phi}^{T} \cdot \mathbf{K}_{\phi}^{-1} \cdot \mathbf{Q}_{\phi}=\mathbf{Q}_{e}^{T} \cdot \mathbf{K}_{e}^{-1} \cdot \mathbf{Q}_{e} .
\end{gathered}
$$

These relations, which hold in all examples discussed in this paper, together with the UCPF form of the conformal characters, motivate our claim that $\mathbf{K}_{e}$ and $\mathbf{K}_{\phi}$ are the appropriate generalizations of the $K$-matrices in this non-Abelian setting.

We shall now proceed and link the composite $Q=-2 e$ particle in the electron sector to the supercurrent that is familiar in the context of the BCS theory for superconductivity. For this argument, it is useful to follow the $q$-Pfaffian state as a function of $q$ with $0 \leqslant q \leqslant 2$. This procedure can be interpreted in terms of a flux-attachment transformation. ${ }^{10,12}$ For $q=2$ we have the fermionic Pfaffian state at $\nu=\frac{1}{2}$ and $q=1$ gives a bosonic Pfaffian state at $\nu=1$. In the nonmagnetic limit, $q \rightarrow 0$, we recognize the Pfaffian wave function as the coordinate space representation of a specific superconducting BCS state with complex $p$-wave pairing. ${ }^{10,12}$ In the limit $q \rightarrow 0$, the particle $\Psi_{2}$ has exclusion parameter $g=0$ and can, as we shall argue, be associated with the supercurrent of the superconducting state. (In fact, the mutual exclusion statistics between $\Psi_{2}$ and $\Psi_{1}$ vanishes as well in the limit $q \longrightarrow 0$.) The exclusion statistics parameter for the $\phi$ particle diverges for $q \rightarrow 0$, meaning that in this limit the $\phi$ sector no longer contributes to the physical edge spectrum.

In a quantum state that has all electrons paired, the fundamental flux quantum is $h / 2 e$. Piercing the sample with precisely this amount of flux leads to a quasihole excitation of charge $e / 2 q$. This excitation also contains a factor that acts as the spin field with respect to the neutral fermion in the electron sector; the latter factor is at the origin of the non-Abelian statistics. ${ }^{6,11}$ For $q=1,2, \ldots$ the quasihole charge $e / 2 q$ is the lowest charge that we consider: the excitations of charge $-e$ and $-2 e$ correspond to flux insertions that are a (negative) integer multiple of the flux quantum. However, for $q \ll 1$, the quasihole charge is larger than $e, 2 e$, and we conclude that the fundamental excitations in the $e$ sector correspond to the insertion of a fraction of the flux quantum, in other words, to a situation where the boundary conditions for the original "electrons" have been twisted.

For definiteness, let us put $q=1 / N$ with $N$ a large integer. A $\Psi_{2}$ quantum state of charge $-2 e$ then corresponds to a flux insertion of $-2 q h / e=-2 h / N e$. In the absence of any $\Psi_{1}$ quanta, this quantum state for the $\Psi_{2}$ particle can be filled up to a maximum of $n^{\max }=1 / 4 q=N / 4$ times. (This follows from the self-exclusion parameter $g=4 q$ and the result that in Haldane's statistics, $n^{\max }=1 / g$.) The amount of 
flux that corresponds to this maximal occupation equals $-(2 h / N e)(N / 4)=-h / 2 e$, which is precisely the flux quantum.

Summarizing, we see that the insertion of a single flux quantum $h / 2 e$ gives rise to a quasihole $(\phi)$ excitation, while (negative) fractions of the flux quantum (between $-h / 2 e$ and $0)$ give rise to multiple occupation of the $\Psi_{2}$ modes.

In the description of a BCS superconductor, the excitation that is induced by the insertion of a fraction of the flux quantum $h / 2 e$ is precisely the supercurrent that screens the imposed flux. Comparing the two pictures, we see that in the limit $q \rightarrow 0$, the $\Psi_{2}$ excitations in the $q$-Pfaffian state reduce to supercurrent excitations in the limiting BCS superconductor. In an earlier study, ${ }^{10}$ the neutral fermionic excitation over the $q$-Pfaffian state [which is not elementary in our $(e, \phi)$ description], in the limit $q \rightarrow 0$, has been identified with the pair-breaking excitation of the superconducting state.

A similar reasoning applies to the Laughlin state at $\nu$ $=1 / m$, where it links the charge $-e$ excitations at finite $m$ to the supercurrent of the limiting superfluid boson state at $m$ $=0$.

In a somewhat different physical picture, the edge particle $\Psi_{2}$ is the one that is excited in the process of Andreev reflection off the edge of a sample in the $q$-Pfaffian state. It will be interesting to explore in some detail such processes for the $q=2$ Pfaffian state.

\section{PSEUDOSPIN-TRIPLET PFAFFIAN QUANTUM HALL STATES}

As a generalization of the results in the previous section, we consider a $q$-Pfaffian state for particles with an internal (double-layer or pseudospin) degree of freedom. The wave function of this state is the Halperin two-layer state with labels $(q+1, q+1, q-1)$. This state has $K$-matrices

$$
\begin{gathered}
\mathbf{K}_{e}=\left(\begin{array}{cc}
q+1 & q-1 \\
q-1 & q+1
\end{array}\right), \\
\mathbf{K}_{\phi}=\frac{1}{4 q}\left(\begin{array}{cc}
q+1 & -q+1 \\
-q+1 & q+1
\end{array}\right),
\end{gathered}
$$

describing excitations $\left(\Psi_{\uparrow}, \Psi_{\downarrow}\right)$ of charge $-e$ and $\left(\phi_{\downarrow}, \phi_{\uparrow}\right)$ of charge $e / 2 q$, respectively. There are no pseudoparticles and these states are Abelian quantum Hall states.

We shall now argue that, based on the analogy with the $q$-Pfaffian states for spinless electrons, for these states we can identify supercurrent-type excitations in the $e$ sector and, by duality, reformulate the $\phi$ sector in terms of one physical quasihole and two pseudoparticles. We stress that this reformulation does not change the physical interpretation; in particular, although the new formulation employs two pseudoparticles, it still refers to an Abelian quantum Hall state.

In the limit $q \rightarrow 0$, the $(q+1, q+1, q-1)$ paired wave function reduces to a form that can be interpreted as a (complex) $p$-wave pseudospin-triplet state. ${ }^{12}$ Taking $q=1 / N$ as before, we can look for excitations in the $e$ sector that describe the response to the insertion of fractional flux, and that will smoothly connect to the supercurrent at $q=0$. Inspecting the matrix $\mathbf{K}_{e}$, we see that the two quanta $\Psi_{\uparrow}$ and $\Psi_{\downarrow}$ each have self-exclusion parameter approaching $g=1$, and by themselves cannot screen more than an amount of flux equal to $h / N e$. However, due to the strong negative mutual exclusion statistics, an excitation that is effectively a pair of one $\Psi_{\uparrow}$ and one $\Psi_{\downarrow}$ particle can screen a much larger amount of flux.

To formalize this consideration, we introduce a particle $\Psi_{3}$, defined as a pair $\left(\Psi_{\uparrow} \Psi_{\downarrow}\right)$. Following the general construction presented in Ref. 9, we derive a new $K$-matrix for the extended system $\left(\Psi_{\uparrow}, \Psi_{\downarrow}, \Psi_{3}\right)$,

$$
\widetilde{\mathbf{K}}_{e}=\left(\begin{array}{ccc}
q+1 & q & 2 q \\
q & q+1 & 2 q \\
2 q & 2 q & 4 q
\end{array}\right),
$$

[This choice of $K$-matrix guarantees an equivalence between the $\left(\Psi_{\uparrow}, \Psi_{\downarrow}\right)$ and $\left(\Psi_{\uparrow}, \Psi_{\downarrow}, \Psi_{3}\right)$ formulations.] On the basis of the extended matrix $\widetilde{\mathbf{K}}_{e}$, we identify the supercurrent excitations as before: a single $\Psi_{3}$ quantum requires flux $2 h / N e$, and with a maximal filling of $n^{\max }=1 / 4 q=N / 4$, we see that the $\Psi_{3}$ quanta can "absorb" an amount of flux equal to $h / 2 e$. In the limit $q \rightarrow 0$, the $\Psi_{3}$ quanta are identified with the supercurrent quanta, which have the ability to screen a full quantum $h / 2 e$ of applied flux. Inverting $\widetilde{\mathbf{K}}_{e}$, we obtain

$$
\widetilde{\mathbf{K}}_{\phi}=\left(\begin{array}{ccc}
1 & 0 & -\frac{1}{2} \\
0 & 1 & -\frac{1}{2} \\
-\frac{1}{2} & -\frac{1}{2} & \frac{2 q+1}{4 q}
\end{array}\right),
$$

with associated parameters

$$
\begin{gathered}
x_{1}=\frac{x_{\downarrow}}{x_{\uparrow}}=\left(\frac{y_{\downarrow}}{y_{\uparrow}}\right)^{1 / 2}, \quad x_{2}=\frac{x_{\uparrow}}{x_{\downarrow}}=\left(\frac{y_{\uparrow}}{y_{\downarrow}}\right)^{1 / 2}, \\
x_{3}=\left(x_{\uparrow} x_{\downarrow}\right)^{1 / 2}=\left(y_{\uparrow} y \downarrow\right)^{-1 / 4 q},
\end{gathered}
$$

where $y_{\uparrow, \downarrow}=\exp \left[\beta\left(\mu_{\uparrow, \downarrow}-\epsilon\right)\right]$. The fact that $x_{1,2}$ do not depend on the energy parameter $\epsilon$ makes clear that these are pseudoparticles. As such they account for degeneracies in states that contain more than one $\phi_{3}$ quantum. Despite this appearance, by construction it is clear that the braid statistics of these degenerate excitations are Abelian.

At the level of the edge CFT, the two different formulations of the $(q+1, q+1, q-1)$ theory are easily understood. In the usual Abelian formulation, the edge CFT is written in terms of a (charge) boson $\varphi_{c}$ plus a Dirac fermion, whose (dimension- $\frac{1}{8}$ ) spin field has Abelian statistics. The alternative formulation employs $\varphi_{c}$ plus two real fermions (called $\psi_{e}$ and $\psi_{o}$ in Ref. 12). The two pseudoparticles that we obtained describe the non-Abelian statistics of the spin fields of the real (Ising) fermions $\psi_{e}$ and $\psi_{o}$ separately. The actual chiral Hilbert space of the edge CFT is, however, a subspace of the Hilbert space of the (Ising) ${ }^{2}$ CFT, and the braid statistics in this subspace are all Abelian.

The various phase transitions described in Ref. 12 are easily traced in the statistics matrices. The transition of the $o$ 
spins into the strong-pairing phase decouples one row of the matrices (3.2) and (3.3), turning them into the matrices (2.6) and (2.3) corresponding to the $q$-Pfaffian state. A subsequent transition of the $e$ spins into the strong-pairing phase leaves only the edge excitations $\Psi_{3}$ and further reduces the matrix to $\mathbf{K}_{e}=4 q$, appropriate for a Laughlin state of charge $-2 e$ particles at filling $\nu=1 / q$.

\section{PARAFERMIONIC QUANTUM HALL STATES: GENERALIZED PAIRING}

In Ref. 7 Read and Rezayi proposed a series of nonAbelian quantum Hall states based on order- $k$ clustering of spinless electrons. The wave functions for these states are constructed with help of the well-known $Z_{k}$ parafermions. The general state of Ref. 7 , labeled as $(k, M)$, has filling fraction $\nu=k /(k M+2)=1 / q$ with $q=M+2 / k$. Fermionic quantum Hall states are obtained for $M$ an odd integer. [For $M=0$ we have a bosonic state with $\mathrm{SU}(2)_{k}$ symmetry.] For $k=1,2$ these new states reduce to the Laughlin $(m=M+2)$ and $q$-Pfaffian $(q=M+1)$ states, respectively.

In Ref. 14, the matrices $\mathbf{K}_{\phi}$ were identified for general $(k$, $M)$. Here we illustrate the $K$-matrix structure for $k=3$, where we have

$$
\begin{gathered}
\mathbf{K}_{\phi}=\left(\begin{array}{ccc}
1 & -\frac{1}{2} & 0 \\
-\frac{1}{2} & 1 & -\frac{1}{2} \\
0 & -\frac{1}{2} & \frac{3 q+1}{-9 q}
\end{array}\right), \\
\mathbf{Q}_{\phi}^{T}=\left(0,0, \frac{e}{3 q}\right),
\end{gathered}
$$

for two pseudoparticles and a physical quasihole of charge $e / 3 q$. Inverting this matrix gives

$$
\begin{gathered}
\mathbf{K}_{e}=\left(\begin{array}{ccc}
q+\frac{2}{3} & 2 q+\frac{2}{3} & 3 q \\
2 q+\frac{2}{3} & 4 q+\frac{4}{3} & 6 q \\
3 q & 6 q & 9 q
\end{array}\right), \\
\mathbf{Q}_{e}^{T}=(-e,-2 e,-3 e) .
\end{gathered}
$$

Again putting $q=1 / N$, with $N$ large, we can repeat the previous arguments. Clearly, the $\Psi_{3}$ quanta of charge $-3 e$ act as the "supercurrent" for the three-electron clustering. One such quantum requires a flux of $-3 h / e N$, and with $n^{\max }$ $=N / 9$ the total flux that can be absorbed equals $-h / 3 e$, as expected.

We remark that, for $q \rightarrow 0$, the excitations $\Psi_{1,2}$ have fractional exclusion statistics parameters, in agreement with the fact that the $k=3$ state at $q=0$ has $M=-\frac{2}{3}$ and is thus not fermionic. What one has instead is an "anyonic"' superconductor with Cooper clusters of charge $-3 e$ and clusterbreaking excitations with fractional exclusion statistics.

\section{NON-ABELIAN SPIN-SINGLET QUANTUM HALL STATES}

In Ref. 8, two of the present authors introduced a series of non-Abelian spin-singlet (NASS) states. The states are labeled as $(k, M)$ and have filling fraction $\nu=2 k /(2 k M+3)$ $=1 / q$ with $q=M+3 / 2 k$. The wave functions, which are constructed as conformal blocks of higher rank (Gepner) parafermions, have a BCS-type factorized form, where the factors describe a $k$-fold spin-polarized clustering of electrons of given spin and the formation of a spin singlet with $2 k$ participating electrons. (See Ref. 8 for an example and Ref. 9 for general and explicit expressions for the wave functions.)

For $k=1$ the spin-singlet states are Abelian with $K$-matrices given by

$$
\begin{gathered}
\mathbf{K}_{e}=\left(\begin{array}{cc}
q+\frac{1}{2} & q-\frac{1}{2} \\
q-\frac{1}{2} & q+\frac{1}{2}
\end{array}\right), \\
\mathbf{K}_{\phi}=\frac{1}{2 q}\left(\begin{array}{cc}
q+\frac{1}{2} & -q+\frac{1}{2} \\
-q+\frac{1}{2} & q+\frac{1}{2}
\end{array}\right) .
\end{gathered}
$$

We remark that, as for the Laughlin series, there is a selfduality in the sense that $\mathbf{K}_{e}(M)=\mathbf{K}_{\phi}\left(M^{\prime}\right)$ with

$$
M=-\frac{3 M^{\prime}+4}{2 M^{\prime}+3} \quad\left(q=\frac{1}{4 q^{\prime}}\right) .
$$

One of the self-dual points is $M=M^{\prime}=-1\left(q=q^{\prime}=\frac{1}{2}\right)$, corresponding to two decoupled $\nu=1$ systems for spin up and down.

In a forthcoming paper ${ }^{9}$ we present a detailed derivation of the $K$-matrix structure for the general NASS states, where we obtain "minimal' $K$-matrices of size $2 k \times 2 k$. The matrix $\mathbf{K}_{e}$ describes fully polarized composites (of both spins) of $1,2, \ldots, k$ quasielectrons, while the matrix $\mathbf{K}_{\phi}$ describes a spin doublet of physical, fractionally charged quasiholes $(Q=e / 4 q)$ and a collection of $2(k-1)$ pseudoparticles that take care of the non-Abelian statistics. The simplest nontrivial example is the result for $k=2\left(M=q-\frac{3}{4}\right)$,

$$
\begin{array}{r}
\mathbf{K}_{\phi}=\left(\begin{array}{cccc}
\frac{4}{3} & \frac{2}{3} & -\frac{2}{3} & -\frac{1}{3} \\
\frac{2}{3} & \frac{4}{3} & -\frac{1}{3} & -\frac{2}{3} \\
-\frac{2}{3} & -\frac{1}{3} & \frac{28 q+3}{48 q} & -\frac{4 q-3}{48 q} \\
-\frac{1}{3} & -\frac{2}{3} & -\frac{4 q-3}{48 q} & \frac{28 q+3}{48 q}
\end{array}\right), \\
\mathbf{K}_{e}=\left(\begin{array}{cccc}
\mathbf{Q}_{\phi}^{T} & =(0,0, e / 4 q, e / 4 q), \\
q+\frac{5}{4} & q-\frac{3}{4} & 2 q+\frac{1}{2} & 2 q-\frac{1}{2} \\
q-\frac{3}{4} & q+\frac{5}{4} & 2 q-\frac{1}{2} & 2 q+\frac{1}{2} \\
2 q+\frac{1}{2} & 2 q-\frac{1}{2} & 4 q+1 & 4 q-1 \\
2 q-\frac{1}{2} & 2 q+\frac{1}{2} & 4 q-1 & 4 q+1
\end{array}\right),
\end{array}
$$




$$
\mathbf{Q}_{e}^{T}=(-e,-e,-2 e,-2 e) .
$$

In analogy with the reasoning for the pseudospin-triplet Pfaffian state, one may now consider the composite of the $k$-spin-up and the $k$-spin-down components, and determine an extended $K_{e}$-matrix [cf. Eq. (3.2)]. For $q \rightarrow 0$ one finds that all statistical couplings of this composite vanish, and we identify it with the supercurrent corresponding to the $2 k$-electron spin-singlet clustering. The extended $K_{e}$-matrix is invertible and gives a redefined $\phi$ sector with a single spinless $\phi$ quantum and $2 k$ pseudoparticles. For obtaining a formulation with manifest $\mathrm{SU}(2)$ spin symmetry, a further extension of $\mathbf{K}_{e}$ can be considered. ${ }^{9}$

\section{CONCLUSIONS}

In this paper, we have studied the exclusion statistics of edge excitations over non-Abelian quantum Hall states. From the results we have extracted matrices $\mathbf{K}_{e}$ and $\mathbf{K}_{\phi}$, which generalize the well-known $K$-matrices for Abelian quantum Hall states to the non-Abelian case. Note, however, that the torus degeneracy for the non-Abelian case is not given by the Abelian result $\left|\operatorname{det}\left(\mathbf{K}_{e}\right)\right|$, but that a further reduction is necessary due to the presence of the pseudoparticles. (Compare with Ref. 17, where such a reduction was discussed in the context of the parton construction of nonAbelian quantum Hall states.)

We expect that these new $K$-matrices can be used to formulate effective (edge and bulk) theories for the non-Abelian quantum Hall states. Until now, effective field theories for bulk excitations (of the LGCS type) have been obtained only for some very special cases, ${ }^{18}$ and it will be most interesting to find more systematic constructions.

\section{ACKNOWLEDGMENTS}

We would like to thank Nick Read for illuminating discussions. This research was supported in part by the Australian Research Council and the foundation FOM of the Netherlands.
${ }^{1}$ X.-G. Wen, Adv. Phys. 44, 405 (1995).

${ }^{2}$ F. D. M. Haldane, Phys. Rev. Lett. 67, 937 (1991).

${ }^{3}$ R. van Elburg and K. Schoutens, Phys. Rev. B 58, 15704 (1998).

${ }^{4}$ A. Berkovich and B. M. McCoy, in Statistical Physics on the Eve of the 21st Century, Advances in Statistical Mechanics, edited by M. T. Batchelor and L. T. Wille (World Scientific, Singapore, 1999), Vol. 14, pp. 240-256.

${ }^{5}$ T. Fukui and N. Kawakami, Phys. Rev. B 51, 5239 (1995).

${ }^{6}$ G. Moore and N. Read, Nucl. Phys. B 360, 362 (1991).

${ }^{7}$ N. Read and E. Rezayi, Phys. Rev. B 59, 8084 (1999).

${ }^{8}$ E. Ardonne and K. Schoutens, Phys. Rev. Lett. 82, 5096 (1999).

${ }^{9}$ E. Ardonne, P. Bouwknegt, and K. Schoutens (unpublished).

${ }^{10}$ M. Greiter, X.-G. Wen, and F. Wilczek, Nucl. Phys. B 374, 567
(1992).

${ }^{11}$ C. Nayak and F. Wilczek, Phys. Rev. Lett. 73, 2740 (1994).

${ }^{12}$ N. Read and D. Green, Phys. Rev. B (to be published).

${ }^{13}$ K. Schoutens, Phys. Rev. Lett. 81, 1929 (1998).

${ }^{14}$ S. Guruswamy and K. Schoutens, Nucl. Phys. B. B556 [FS], 530 (1999).

${ }^{15}$ S. B. Isakov, Mod. Phys. Lett. B 8, 319 (1994); S. Ouvry, Phys. Rev. Lett. 72, 600 (1994); Y.-S. Wu, ibid. 73, 922 (1994).

${ }^{16}$ P. Bouwknegt, L. Chim, and D. Ridout, Nucl. Phys. B (to be published).

${ }^{17}$ X.-G. Wen and A. Zee, Phys. Rev. B 58, 15717 (1998).

${ }^{18}$ E. Fradkin, C. Nayak, and K. Schoutens, Nucl. Phys. B 546, 711 (1999). 